01

\title{
Влияние термополевой эмиссии электронов на формирование аморфных металлических наночастиц в плазме лазерного факела
}

\author{
(С) А.А. Борматов, В.М. Кожевин, С.А. Гуревич \\ Физико-технический институт им. А.Ф. Иофрфе РАН, \\ 194021 Санкт-Петербург, Россия \\ e-mail: antonbormat@mail.ru
}

Поступило в Редакцию 5 октября 2020 г.

В окончательной редакции 9 декабря 2020 r.

Принято к публикации 10 декабря 2020 г.

В последние годы значительно усилился интерес к использованию структур, состоящих из аморфных наночастиц, который обусловлен их необычными электрическими, магнитными и каталитическими свойствами. Одной из важных задач, стоящих на пути к практическому применению таких структур, является разработка новых технологий их формирования. Исследования, проведенные в рамках решения этой задачи, показали, что наиболее эффективным способом получения аморфных металлических наночастиц является дробление микрокапель металлов, заряжаемых в плазме лазерного факела. Однако, несмотря на то, что использование этого метода позволило создать такие структуры из различных типов металлов, теоретический анализ эффекта дробления микрокапель проведен без детального рассмотрения процесса зарядки нанометровых капель, что в значительной мере ограничивает возможность использования имеющихся теоретических моделей для разработки новых технологий. Представлена модель, позволяющая более точно определить требования к параметрам плазмы лазерного факела, при которых реализуется эффективное дробление микрокапель металлов до нанометровых размеров.

Ключевые слова: термополевая эмиссия, плазма лазерного факела, аморфные наноструктуры, предел Рэлея, каскадное электродиспергирование.

DOI: $10.21883 / J T F .2021 .05 .50682 .283-20$

\section{Введение}

Существенные отличия свойств аморфных металлов, называемых также металлическими стеклами, от свойств кристаллических аналогов известны давно [1]. К наиболее важным особенностям аморфных металлов, определяющим их широкое применение в прикладных целях, следует отнести как их повышенную прочностную и коррозионную стойкость [2], так и их необычные магнитные и каталитические свойства [3]. Однако возможности расширения областей применения металлических стекол ограничиваются тем, что для перевода металлов в аморфное состояние необходимо обеспечить резкое охлаждение расплавов в момент их затвердевания. Особенно большие трудности возникают при попытках перевода в аморфное состояние чистых металлов, поскольку требуемая для этого скорость охлаждения расплава варьируется в пределах от $10^{9}$ до $10^{13} \mathrm{~K} \cdot \mathrm{s}^{-1}$ [4].

Обеспечить отвод тепла, при котором реализуются такие высокие скорости охлаждения, можно только за счет резкого уменьшения характерного размера расплава. В реальных условиях существует два способа снижения этого размера. В первом из них на поверхности металлов с высокой теплопроводностью формируются ультратонкие пленки расплава, что приводит к аморфизации поверхности этих металлов [5]. Второй способ, используемый для создания объемных металлов с аморфной структурой, основан на дроблении расплавов на множество мелких капель с дальнейшим их осаждением после затвердевания [6]. Рассматриваемый в настоящей работе метод аморфизации металлов основан на использовании процесса второго типа, т. е. дробления расплава.

Эксперименты по исследованию возможности дробления металлов под воздействием внешних сил показали, что некоторые чистые металлы, у которых требуемая скорость охлаждения расплава не превышает $10^{10} \mathrm{~K} \cdot \mathrm{s}$, могут быть переведены в аморфное состояние, если финальный размер капель снижается до 200-300 nm [6]. Однако для перевода в аморфное состояние большинства чистых металлов необходимо повысить скорости охлаждения расплавов на два-три порядка, что, в свою очередь, требует снижения размеров капель до нескольких нанометров. Реализация такого дробления капель под воздействием внешних сил практически невозможна, поскольку при уменьшении размеров капель до нанометровых масштабов катастрофически возрастают капиллярные силы.

В соответствии с вышесказанным единственной возможностью реализации дробления капель расплава до более мелких размеров является использование методик, позволяющих снизить влияние капиллярных сил. Такое снижение может быть осуществлено за счет зарядки капель, которая приводит появлению на их поверхности отрицательного давления электрического поля, компенсирующего давление поверхностного натяжения расплава, что может привести к переходу капель в неустойчивое состояние и, как следствие, к их делению. 


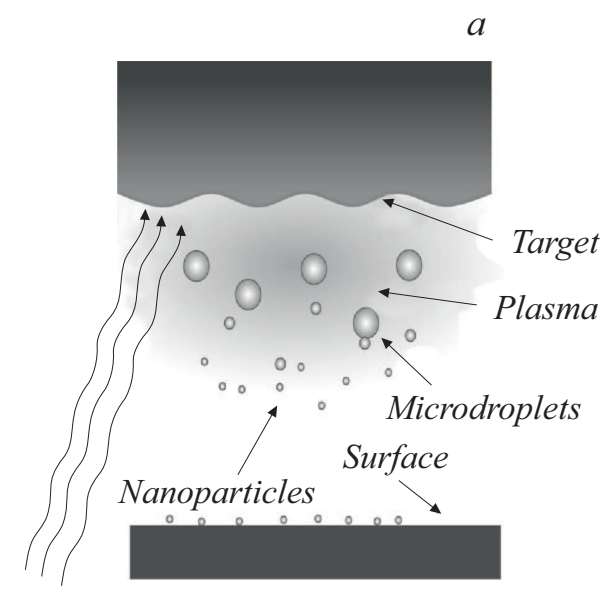

$b$

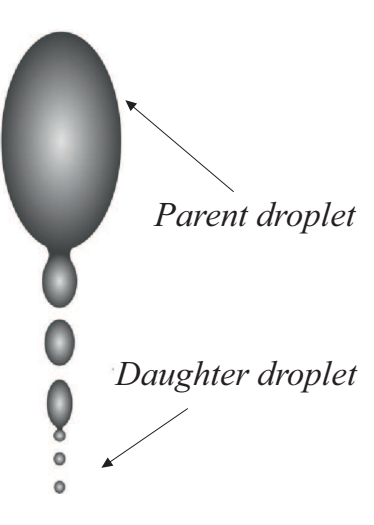

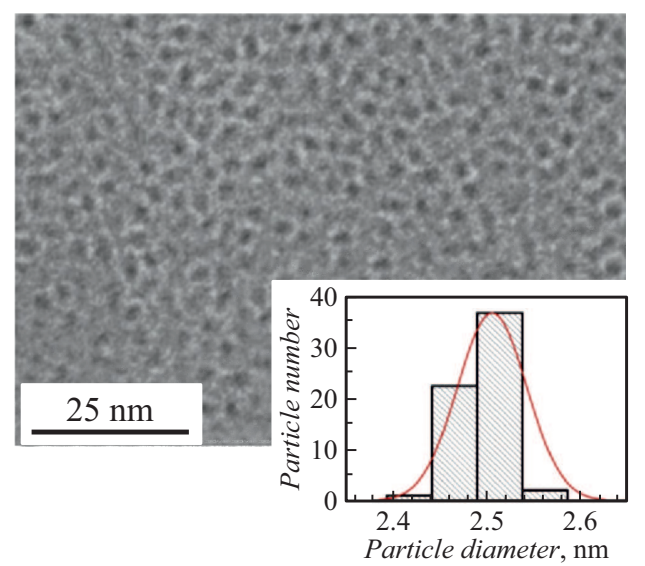

Рис. 1. $a-$ схема метода лазерного электродиспергирования. Волнистыми линиями изображен поток лазерного излучения на поверхность мишени; $b-$ схема каскадного деления капли; $c-$ TЭМ изображение поверхности подложки с Ni наночастицами, полученных методом лазерного электродиспергирования [19].

Теоретические и экспериментальные исследования влияния на устойчивость капель отрицательного давления, обусловленного их зарядкой, проводятся, начиная с работы Рэлея, опубликованной в 1882 г. и переизданной позднее в более удобном для чтения виде [7]. В этой работе было показано, что волны на поверхности капель становятся неустойчивыми и капли начинают делиться, когда их заряд превышает критическое значение, называемое пределом Рэлея, которое равно

$$
Q_{R}=8 \pi \sqrt{\varepsilon_{0} \alpha R^{3}} .
$$

Здесь $R$ - радиус капли, $\alpha$ - коэффициент поверхностного натяжения, а $\varepsilon_{0}$ - электрическая постоянная. Отметим также, что при заряде сферической капли, равном пределу Рэлея, давление электрического поля на поверхности капель полностью компенсирует давление сил поверхностного натяжения. Более поздние исследования как теоретические $[8]$, так и экспериментальные $[9,10]$ подтвердили факт распада капель при превышении зарядом предела Рэлея, причем было показано, что этот распад имеет каскадный характер.

Схематически последовательные ступени такого распада показаны на рис. 1, из которого видно, что перезаряженная материнская капля эмитирует большое количество более мелких дочерних капель, размер которых во много раз меньше размера материнской капли. При этом важно, что дочерние капли также перезаряжены в смысле предела Рэлея [8] и эмитируют дочерние капли следующего поколения, вследствие чего процесс становится каскадным. В работе [8] полагалось, что такой характер каскадного деления сохраняется при всех размерах частиц, и в финальной стадии такого деления могут формироваться капли с размером вплоть до нанометров. В то же время из результатов более поздних оценок, представленных, например, в [11,12], следовало, что основной причиной остановки процесса деления является резкий рост интенсивности эмиссии электронов с поверхности капель, заряженных до предела Рэлея, который возникает при уменьшении их размеров этих капель до нескольких нанометров.

Обнаружение эффекта распада сильно заряженных капель обусловило развитие многочисленных приложений, направленных на производство мелкодисперсных сред. В качестве примеров можно привести использование этого явления в жидкометаллических источниках ионов [13], в процессах жидкометаллической эпитаксии и литографии [14], при получении порошков тугоплавких металлов [15], в реактивной космической технике [16], в ускорителях макрочастиц [17].

Однако все упомянутые методы малопригодны для формирования наноструктур, поскольку в окончательных продуктах деления массовая доля наночастиц ничтожно мала. Основная причина этого состоит в том, что в процессе каскадного деления большая доля заряда материнских капель уносится дочерними каплями, при этом в упомянутых технологиях не реализован процесс подзарядки капель во время их деления и все капли заряжаются лишь однажды. Соответственно на каждом этапе каскадного процесса деление материнских капель прекращается при сбросе ими небольшой доли своей массы. Таким образом, для повышения эффективности процесса дробления капель расплава, необходимо разрабатывать методы, позволяющие осуществлять постоянную подзарядку материнских капель на всех этапах деления.

Такой метод, называемый методом лазерного электродиспергирования, был представлен в работах $[11,12]$. Основной особенностью этого метода является то, что зарядка и деление капель расплава происходят при погружении этих капель в плотную плазму лазерного факела, вследствие чего подзарядка этих капель происходит постоянно. Привлекательной особенностью метода является также то, что инжекция микрокапель в плазму 
происходит автоматически вследствие выплеска этих капель с поверхности расплава облучаемой мишени. Выброс капель с поверхности расплава происходит одновременно с формированием контактирующей с ним плазмы, поскольку выплескивание капель является следствием развития капиллярной неустойчивости расплава, возбуждающейся под воздействием плазмы.

Экспериментальные исследования показали, что металлические наноструктуры, формируемые с использованием этого метода, состоят из аморфных наночастиц с размером 2-5 nm, обладающих повышенной стойкостью к коагуляции и окислению [12]. Было также показано, что эти наноструктуры обладают необычными электрическими, магнитными, каталитическими и оптическими свойствами [18-21].

Теоретическое описание процессов зарядки капель, представленное в работах $[11,12]$, проводилось с использованием модели, в которой учитывались практически все основные эффекты. Однако построение этой модели, предназначенной для аналитического описания рассматриваемых процессов, основывалось на достаточно жестких предположениях. Так, заряженный слой, всегда формирующийся у поверхности металлических капель в плазме и называемый плазменным слоем или слоем Ленгмюра, описывался в рамках модели сферического конденсатора с расстоянием между обкладками равным величине дебаевской длины в плазме. Такое приближение позволяет создать простую модель, позволяющую провести аналитические оценки, однако границы применимости этой модели до конца не определены. Кроме того, анализ эмиссии электронов с поверхности капель проводился только в рамках полевой эмиссии, т.е. высокая температура, характерная для капель металла в плотной лазерной плазме, не учитывалась. Соответственно использование модели, представленной в $[11,12]$ позволяет проводить только качественные оценки процессов зарядки капель в плазме.

В связи с этим основными целями настоящей работы являются как разработка более точной модели, позволяющей проводить количественный анализ процесса зарядки капель в плазме, так и проведение расчетов, направленных на уточнение требований к параметрам плазмы, при которых реализуется эффективная зарядка капель в широком диапазоне их размеров.

\section{1. Моделирование процесса зарядки капель в плазме лазерного факела}

Рассмотрим более подробно постановку задачи о моделировании процесса зарядки капель в плазме лазерного факела. Отметим, прежде всего, что вследствие разлета плазмы параметры ее являются нестационарными. В связи с этим, в общем случае предполагается, что для описания всех процессов, происходящих в плазме лазерного факела, следует использовать нестационарные модели. Заметим, однако, что интенсивность начальной зарядки капель, инжектированных в плазму, определяется, прежде всего, потоком электронов на ее поверхность. При этом она чрезвычайно велика, что делает время зарядки капель экстремально коротким по сравнению с характерным временем изменения параметров плазмы. Поэтому заряд капли является функцией плотности и температуры плазмы в текущий момент времени. Рассмотрим ограничения на параметры плазмы, при которых возможно использовать стационарную модель зарядки капли.

Капли металлов, как и все тела, помещенные в плазму, прекращают заряжаться, когда разность потенциалов между заряженной каплей и плазмой достигает предельной величины $U_{f l}$, называемой плавающим потенциалом. Этот эффект обусловлен тем, что плотность тока электронов на каплю, описываемая формулой

$$
J_{e}=e n \sqrt{k T_{e} / m_{e}} \exp \left(-\frac{e U}{k T_{e}}\right),
$$

уменьшается при увеличении потенциала $U$ и соответствующем возрастании заряда капли, который равен $Q=C_{d} U$. Здесь $n-$ плотность плазмы, $m_{e}$ и $T_{e}-$ масса и температура электронов, $C_{d}$ - емкость капли в плазме. Снижение плотности тока электронов на каплю продолжается до того момента, когда она уменьшается до величины, при которой ионный и электронный токи становятся равными. Плавающий потенциал, соответствующий этому балансу токов, равен

$$
U_{f l} \approx\left(k T_{e} / e\right) \ln \left(m_{i} / m_{e}\right)^{1 / 2},
$$

где $m_{i}$ и $m_{e}$ - массы ионов и электронов плазмы соответственно, $e-$ заряд электрона, $k-$ постоянная Больцмана. Как видно из (2), при фиксированной температуре электронов плавающий потенциал $U_{f l}$ постоянен. Важно также отметить, что потенциал капель не может превысить $U_{f l}$, вследствие чего заряженными до предела Рэлея могут быть только капли, размер которых с учетом формулы (1) удовлетворяет неравенству $\varepsilon_{0} \alpha R^{3} \leq\left(C_{d} U_{f l} / 8 \pi\right)^{2}$.

Учитывая, что соотношение (2) справедливо только в случае, когда характерное время первоначального заряжения капель до плавающего потенциала существенно меньше времени изменения параметров плазмы, проведем оценку времени первоначальной зарядки капель из решения уравнения $d Q / d t=J_{e}$, которое имеет вид

$$
C_{d} \frac{d U}{d t}=4 \pi R^{2} e n \sqrt{k T_{e} / m_{e}} \exp \left(-\frac{e U}{k T_{e}}\right)
$$

Отметим, что уравнение (3) записано в упрощенном виде, а именно, в нем учитывается только зарядка капель под воздействием электронного тока из плазмы и соответственно пренебрегается вкладами в этот процесс других потоков, определяющих заряд, таких как поток ионов и эмиссионный поток электронов с поверхности капель. Рассмотрим основные аргументы в пользу такого подхода. 
Пренебрежение влиянием тока ионов обусловлено тем, что в основной стадии зарядки капель, т.е. при зарядке их от нейтрального состояния до заряда, соответствующего потенциалу $U \approx 0.8 U_{f l}$, ток электронов существенно превосходит ток ионов. Учитывая, что нас интересует зарядка капель только до предела Рэлея $Q_{R}$, т. е. до потенциала $U_{R}$, который, как указывалось выше, всегда меньше $U_{f l}$, можно считать, что током ионов в этой стадии можно пренебречь практически всегда.

Эмиссионный поток электронов с поверхности капель может определяться в общем случае эффектами их вторичной и термополевой эмиссий. Что касается вторичной эмиссии электронов, то она становится существенной только при энергии электронов более $100 \mathrm{eV}$, когда коэффициент вторичной эмиссии становится близким к единице. Однако в настоящей работе рассматриваются режимы лазерной абляции с температурой электронов, не превышающей $20 \mathrm{eV}$, и соответственно можно считать влияние вторичной эмиссии ничтожным.

Необходимым условием генерации потоков термополевой эмиссии электронов с поверхности капель, которые сравнимы с потоком электронов из плазмы, является наличие на их поверхности электрического поля с напряженностью, превышающей $2 \cdot 10^{9} \mathrm{~V} / \mathrm{m}$. Такие поля, в частности, характерны для капель нанометрового размера, заряженных до предела Рэлея. Соответственно учет термополевой эмиссии необходим при анализе заряда нанокапель и ее влияние на эффективность зарядки капель до предела Рэлея подробно анализируется в настоящей работе. Однако следует учитывать также, что нанометровые капли являются продуктом деления более крупных капель, и, как показано в [8], с момента их формирования перезаряжены в смысле достижения предела Рэлея. В этом случае стадия первоначального заряжения таких капель, описываемая уравнением (3), просто отсутствует и учет термополевой эмиссии не является необходимым. Таким образом, оценки показывают, что приближения, используемые при выводе уравнения (3), вполне оправданы при оценке времени их зарядки.

Время зарядки капель до предела Рэлея, найденное из решения уравнения (3), с учетом (2) описывается формулой

$$
\tau_{U_{f l}} \approx \frac{\sqrt{m_{i} k T_{e}} C_{d}}{4 \pi R^{2} e^{2} n} .
$$

Емкость капель в плазме в рамках модели сферического конденсатора с расстоянием между обкладками $2 R_{D}$ (здесь $R_{D}$ - дебаевская длина) равна $C_{d}=$ $=2 \pi \varepsilon_{0} R\left(R+2 R_{D}\right) / R_{D}$. Выбор такого расстояния между обкладками обусловлен тем, что, как будет показано далее из результатов численного счета, оно является типичным для ширины заряженного слоя, формирующегося на границе плазмы и капли. В этом случае при $R \geq R_{D}$ эта формула принимает вид $\tau_{U_{f l}} \approx$ $\approx 0.5 \sqrt{m_{i} \varepsilon_{0} / e^{2} n}$. В соответствие с полученной оценочной формулой время зарядки капель не превосходит $10^{-10} \mathrm{~s}$ при плотности плазмы большей, чем $10^{20} \mathrm{~m}^{-3}$.
Сравнение этого времени с характерным временем изменения параметров плазмы лазерного факела, типичная скорость разлета которого близка к $10^{4} \mathrm{~m} / \mathrm{s}$, показывает, что зарядка наночастиц происходит во много раз быстрее, чем изменяются параметры плазмы. Таким образом, проведенные оценки показали, что равновесное распределение заряда и потенциала в слое Ленгмюра, формирующегося на границе капли и плазмы, можно описывать в рамках квазистационарной модели.

Для нахождения распределения потенциала в слое Ленгмюра необходимо решить уравнение Пуассона, которое в приближении сферически симметричной капли имеет вид

$$
\frac{1}{r^{2}} \frac{d}{d r}\left(r^{2} \frac{d U}{d r}\right)=\frac{e}{\varepsilon_{0}}\left(n_{i}-n_{e}\right),
$$

где $U$ - электрический потенциал внутри слоя, а $n_{i}$ и $n_{e}-$ плотности ионов и электронов в слое. Уравнение (5) является уравнением второго порядка, поэтому его необходимо дополнить двумя граничными условиями (см. рис. 2). На поверхности капли выполняется условие стационарности заряда, которое обеспечивается балансом токов заряженных частиц

$$
J_{i}\left(U_{d}\right)+J_{e m}\left(E_{d}\right)-J_{e}\left(U_{d}\right)=0,
$$

где $J_{i}$ и $J_{e m}$ - потоки ионов и эмиссионных электронов соответственно, $U_{d}=\left.U\right|_{r=R}$ и $E_{d}=\left.(d U / d r)\right|_{r=R}-$ потенциал и напряженность электрического поля на поверхности капли соответственно. Другими словами, условие (6) показывает, что ток электронов в стационарном случае всегда скомпенсирован суммой ионного и эмиссионного токов. Отметим, что ток термополевой эмиссии электронов зависит от напряженности электрического поля на поверхности капли. Это приводит к тому, что в случае учета этого тока условие (6), являющееся граничным условием для уравнения (5), становится нелинейным.

На границе слоя с плазмой выполняются условия квазинейтральности

$$
\begin{aligned}
& U_{p l}=0, \\
& E_{p l}=0 .
\end{aligned}
$$

Условие $U_{p l}=0$ служит вторым граничным условием для уравнения (5). Дополнительное граничное условие $E_{p l}=0$ используется для определения ширины плазменного слоя $L$. Таким образом, в общем случае задача (5)-(7) является нелинейной задачей с неопределенной границей. Однако в предположении о возможности использования сферически симметричной модели ситуация существенно упрощается, поскольку при этом становятся доступными достаточно простые численные методы, например, метод „пристрелки“ [22].

Для конкретизации уравнений (5)-(7) необходимо знать распределение плотностей и потоков заряженных частиц в слое. В рамках используемой модели распределение плотности потока ионов в слое вычисляется 

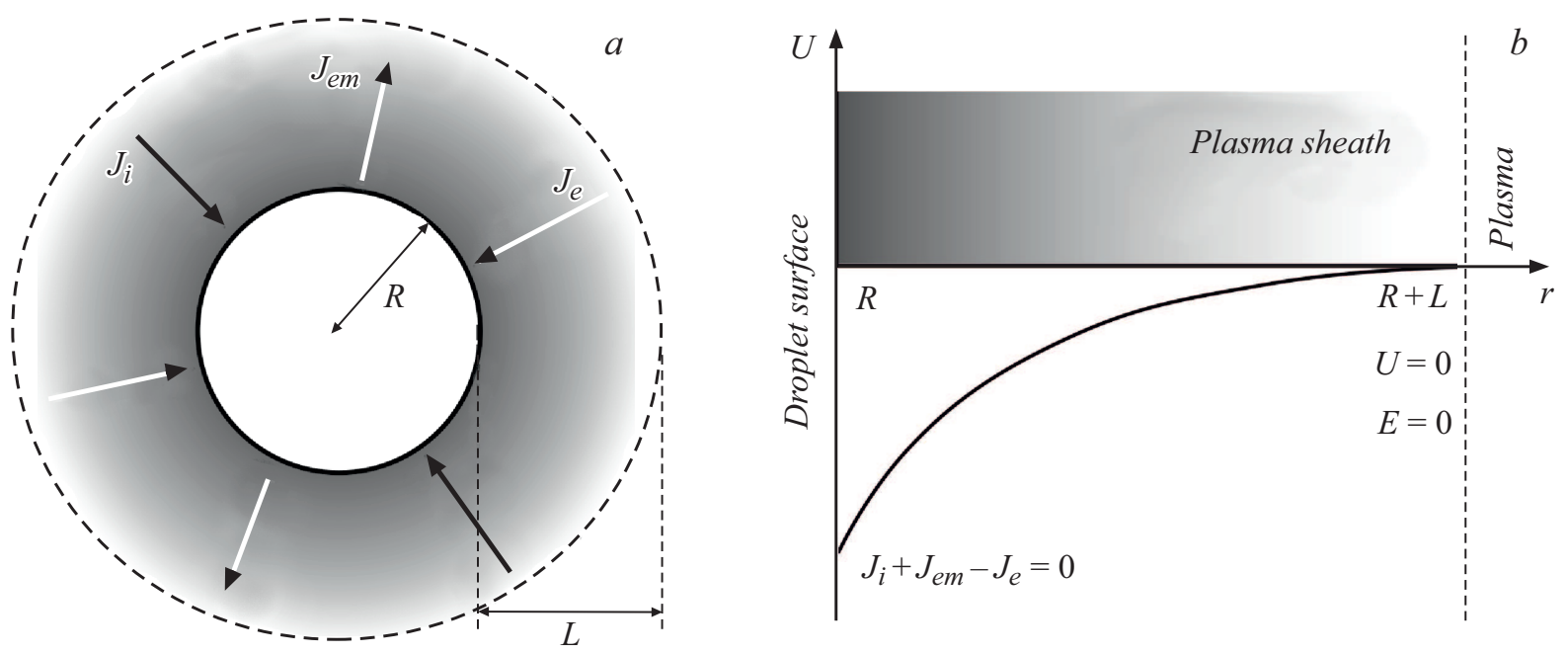

Рис. 2. Модель зарядки капли в плазме: $a-$ схема потоков заряженных частиц на поверхность капли; $b-$ схематичное распределение потенциала электрического поля в слое и граничные условия для уравнения (5).

по формуле Ленгмюра, использующей приближение максвелловского распределения ионов по скоростям в момент их влета в слой [23]

$$
J_{i}=e n \sqrt{\frac{k T_{e}}{m_{i}}}\left[s^{2}+\left(1-s^{2}\right) \exp \left(\frac{e U}{k T_{e}\left(1-s^{2}\right)}\right)\right] .
$$

Здесь $s=1+R_{D} / r, R_{D}$ - дебаевский радиус плазмы. Поток ионов на поверхности капли в этом случае вычисляется с помощью формулы (8), при $U=U_{d}$ и $r=R$. С использованием этой формулы можно получить плотность ионов, учитывая их ускорение при прохождении разности потенциалов в слое:

$n_{i}=\frac{J_{i}}{e u_{i}}=\frac{n}{\sqrt{1+\frac{2 e U}{k T_{e}}}}\left[s^{2}+\left(1-s^{2}\right) \exp \left(\frac{e U}{k T_{e}\left(1-s^{2}\right)}\right)\right]$,

где $u$ - скорость ионов в слое, которая находится из соотношения $m_{i} u_{i}^{2} / 2-e U=k T_{e}$, выражающего закон сохранения энергии для иона в электрическом поле. Отметим также, что при выводе формул (8), (9) учитывалось, что плазменный слой окружен областью предслоя, в котором ионы ускоряются до ионно-звуковой скорости $u_{p l}=\sqrt{k T_{e} / m_{i}}$ под воздействием гидродинамического давления [24].

Плотность эмиссионных электронов в слое существенно меньше плотности электронов плазмы, так как даже в предельном случае равенства потока электронов эмиссионному потоку скорость электронов плазмы изза торможения в слое существенно ниже скорости эмиссионных электронов. Поэтому в правой части уравнения (5) вкладом эмиссионных электронов в заряд слоя можно пренебречь. При этом плотность электронов плазмы достаточно точно может быть описана распределением Больцмана [24]

$$
n_{e}=n \exp \left(-\frac{e U}{k T_{e}}\right)
$$

Соответствующее выражение для потока электронов на поверхности капли имеет вид $J_{e}=n \sqrt{k T_{e} / m_{e}} \times$ $\times \exp \left(-e U_{d} / k T_{e}\right)$. Несмотря на возможность пренебрежения потоком эмиссионных электронов в уравнении (5), в граничном условии (6) этим током пренебрегать нельзя, поскольку для нанокапель он может существенно превышать поток ионов.

Термополевой эмиссионный поток определяется потоком электронов, прошедших через потенциальный барьер, всегда существующий на поверхности капли. Для описания потока электронов, вылетающих на поверхность из объема капли, обычно используют модель свободных электронов Зоммерфельда. В рамках этой модели поток электронов с энергией, лежащей в диапазоне $[W ; W+d W]$, может быть представлен в виде [25]

$$
N(W) d W=z_{S} k T_{s} \ln \left(1+\exp \left[-\frac{W-W_{F}}{k T_{s}}\right]\right) d W,
$$

где $z_{S}=4 \pi m_{e} h^{-3}-$ константа Зоммерфельда, $T_{s}$ температура поверхности капли, $W_{F}$ - энергия Ферми металла, $k$ - постоянная Больцмана. Тогда поток эмитированных электронов рассчитывается по формуле

$$
J_{T F}=\int_{0}^{\infty} N(W) D(W) d W
$$

где $D(W)$ - коэффициент прозрачности потенциального барьера для электрона с энергией $W$, который может быть найден с помощью численного решения уравнения Шредингера [26].

Значение коэффициента прозрачности $D(W)$ зависит от формы потенциального барьера, работы выхода металла $w$ и напряженности внешнего электрического поля на поверхности капли $E_{d}$. Работа выхода металла характеризует высоту барьера, которая снижается под действием поля со значения $w$ до $w-c_{S} \sqrt{E_{d}}$, где 

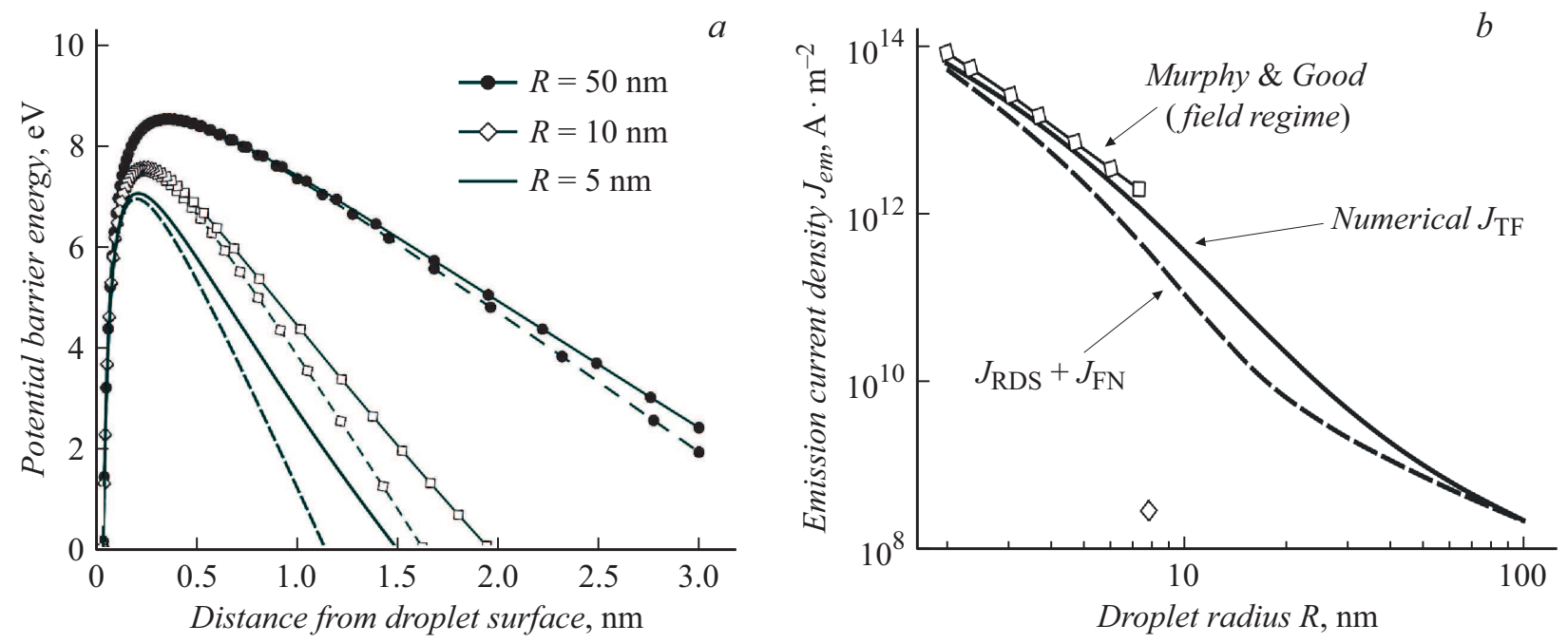

Рис. 3. Термополевая эмиссия с поверхности капли, заряженной до предела Рэлея. $a$ - потенциальный барьер для электрона вблизи поверхности капли. Сплошная кривая получена с учетом сферичности капли, штриховая - в приближении плоской поверхности. $b$ - сравнение результатов расчета плотности тока термополевой эмиссии электронов с использованием аналитических и численных моделей.

$c_{S}=\left(e^{3} / 4 \pi \varepsilon_{0}\right)^{1 / 2}-$ константа Шоттки. Кроме этого, воздействие электрического поля приводит к уменьшению ширины потенциального барьера. Обычно при проведении расчетов термополевой эмиссии с поверхности наночастиц учитывается влияние кривизны этих частиц на форму барьера. Так, в работе [27] показано, что влияние сферичности капли приводит к уширению барьера, и, как следствие, к существенному снижению эмиссионного потока с поверхности капель радиусом в несколько нанометров. Однако в частном случае капель, заряженных до предела Рэлея, напряженность электрического поля на поверхности которых равна

$$
E_{R}=\frac{Q_{R}}{4 \pi \varepsilon_{0} R^{2}}=2 \sqrt{\frac{\alpha}{\varepsilon_{0} R}},
$$

влияние сферичности практически полностью компенсируется сжатием барьера за счет действия внешнего поля (см. рис. $3, a)$. Это позволяет при моделировании процесса зарядки таких нанокапель пренебречь влиянием сферичности на форму потенциального барьера.

Численный метод, несмотря на высокую точность, имеет в качестве главного недостатка резко возрастающие временные затраты при расчете заряда капли. Поэтому в настоящей работе были проанализированы некоторые известные аналитические аппроксимации интеграла (12) в интересующем диапазоне напряженности электрического поля $E_{d}=2-10 \mathrm{GV} / \mathrm{m}$ и температуры поверхности капли $T_{s}=1000-3000 \mathrm{~K}$.

Наиболее простая аналитическая аппроксимация интеграла (12) находится с помощью представления эмиссионного потока электронов в виде суммы двух потоков: электронов, прошедших над барьером, и электронов, туннелировавших сквозь барьер. Поток надбарьерных электронов определяет температурную составляющую эмиссии и, если эффектом отражения электронов от потенциального барьера пренебречь, соответствующая плотность тока может быть описана формулой Ричардсона-Дешмана с поправкой Шоттки [28]

$$
J_{R D S}=A_{R} T_{s}^{2} \exp \left(-\frac{w-c_{s} \sqrt{E_{d}}}{k T_{s}}\right),
$$

где $A_{R}=z_{s} k^{2}-$ константа Ричардсона, $w-$ работа выхода металла. Вклад электронов, туннелирующих через барьер, с поверхности металла при низких температурах описывается законом Фаулера-Нордгейма [29]

$$
J_{F N}=\frac{a_{F N} E_{d}^{2}}{w t^{2}(y)} \exp \left(-\frac{b_{F N} w^{3 / 2} v(y)}{E_{d}}\right) .
$$

Здесь $a_{F N}$ и $b_{F N}-$ константы Фаулера-Нордгейма, $t(y)$ и $v(y)$ - эллиптические функции, которые зависят от величины $y=c_{S} \sqrt{E_{d}} / w$ и легко вычисляются с использованием аналитической аппроксимации, представленной в работе [30]. Таким образом, наиболее простой способ вычисления термополевой эмиссии заключается в расчете суммы $J_{R D S}+J_{F N}$. Среди других известных аппроксимаций наилучшую применимость для нанометрового размера капель показала формула Мерфи-Гуда для полевого режима термополевой эмиссии [31].

Сравнение результатов расчетов термополевой эмиссии по аналитическим аппроксимациям с численным расчетом интеграла (12) приведено на рис. $3, b$. Анализ показал, что применение суммы потоков $J_{R D S}+J_{F N}$ для расчета эмиссии имеет смысл только для проведения предварительных оценок. Слабая применимость вышеупомянутых аналитических формул связана с тем, что эмиссия наиболее хорошо исследована только в областях с преобладанием либо температурной составляющей потока (надбарьерный поток), либо полевой (туннелировавшим потоком электронов). В промежуточной 
области, в которой одновременно существенный вклад вносят оба потока, термополевая эмиссия чаще всего описывается с использованием численных методов. В связи с этим в настоящей работе был выбран наиболее общий и точный способ расчета $J_{T F}$, основанный на численном расчете интеграла (12).

Таким образом, модель зарядки капли в плазме лазерного факела, представленная в настоящей работе, основана на решении уравнения (5) с учетом граничных условий (6)-(7) и формул для токов и плотностей заряженных частиц плазмы $(8)-(10)$ и плотности тока термополевой эмиссии (12), причем коэффициент прозрачности барьера $D(W)$ в формуле (12) рассчитывается на основе уравнения Шредингера. Найденное из решения уравнения (5) распределение потенциала в слое позволяет найти напряженность электрического поля на поверхности капли $E_{d}$ и вычислить заряд капли по формуле $Q=4 \pi \varepsilon_{0} R^{2} E_{d}$. Учитывая, что предложенная модель является достаточно общей, ее использование позволяет, вообще говоря, проводить расчеты заряда капель для широкого диапазона их материалов капель и параметров плазмы. Тем не менее существуют ограничения на использование этой модели, как при высокой температуре плазмы, так и при высокой ее плотности. Так, повышение температуры плазмы приводит к увеличению коэффициента вторичной электронной эмиссии и к необходимости учета этой эмиссии при расчете заряда, т. е. применение модели, как отмечалось выше, возможно только при $T_{e} \ll 100 \mathrm{eV}$. Кроме того, при повышении плотности плазмы выше $n>10^{25} \mathrm{~m}^{-3}$ ширина плазменного слоя, которая пропорциональна дебаевскому радиусу, становится сравнимой с шириной потенциального барьера на поверхности металла. Это приводит к искажению барьера из-за пространственного распределения потенциала в слое и необходимости дальнейшей модификации модели с целью учета квантомеханических эффектов при описании потоков частиц на поверхность капли.

\section{2. Результаты расчетов и обсуждение}

Построенная стационарная модель зарядки капель позволяет рассчитать ее заряд $Q$ для различных материалов и параметров плазмы. Анализируя на основании этих расчетов условия, при которых относительный заряд капель $Q / Q_{R}$ превышает единицу, можно сформулировать требования к параметрам плазмы, при которых реализуется процесс каскадного деления этих капель. Отметим, что относительный заряд капель зависит как от параметров плазмы (плотности, температуры и массы ионов), так и от параметров капель (радиус капель, работа выхода, температура поверхности, энергия Ферми, коэффициент поверхностного натяжения). Поэтому при проведении расчетов важным моментом является выбор значений этих параметров.

В связи с тем, что целью настоящей работы является уточнение требований к параметрам плазмы, при которых в широком диапазоне размеров капель реализуется их зарядка до предела Рэлея, варьируемыми расчетными параметрами были выбраны плотность и температура электронов плазмы, а также радиус капель. Параметры плазмы при расчетах заряда варьировались в характерном для плазмы лазерного факела диапазоне $n \sim 10^{23}-10^{25} \mathrm{~m}^{-3}, T_{e} \sim 10-20 \mathrm{eV}$. Радиус капель $R$ варьировался в диапазоне от $1 \mu \mathrm{m}$ до нескольких нанометров. Параметры материала в большинстве расчетов имели модельные значения, характерные для многих металлов [32]: работа выхода $w=5.0 \mathrm{eV}$, коэффициент поверхностного натяжения $\alpha=1 \mathrm{~N} / \mathrm{m}$. Температура поверхности капель принимала значение, близкое к температуре кипения расплавов.

Для оценки влияния эффектов, учтенных в представленной выше модели, расчеты заряда капли были проведены с использованием трех типов моделей. Целью использования этих моделей было сравнение результатов, полученных с использованием аналитической модели, представленной в работе [12], численной модели без учета термополевой эмиссии, а также численной модели с учетом термополевой эмиссии. Напомним, что преимущество аналитической модели, учитывающей влияние плазменного слоя в рамках модели сферического конденсатора, с шириной между обкладками, равной радиусу Дебая $R_{D}$, определяется возможностью вычисления относительного заряда капель $Q / Q_{R}$ по простой формуле

$$
\frac{Q}{Q_{R}}=\frac{\sqrt{\varepsilon_{0} R}}{4 \sqrt{\alpha}} \frac{k T_{e}}{e} \ln \left(\frac{m_{i}}{m_{e}}\right)\left[\frac{1}{R}+\frac{1}{R_{D}}\right],
$$

в то время как численные модели не представляют такой возможности, но являются более точными.

Результаты проведенных расчетов приведены на рис. 4, $a$. В первую очередь сравнение результатов, полученных с учетом эмиссии электронов (сплошная кривая) и без учета эмиссии (штрихпунктирная кривая) показывает, что эмиссия электронов для крупных капель, радиус которых больше $100 \mathrm{~nm}$, не вносит существенного вклада в заряд капли. Таким образом, процесс зарядки капель в плазме можно достаточно четко разделить на два режима: эмиссионный и безэмиссионный.

Для анализа безэмиссионного режима зарядки капель сравним результаты, полученные из численного расчета заряда без учета эмиссии (штрихпунктирная кривая) и аналитической модели, представленной в работе [12] (штриховая кривая). Заметим, что эти кривые имеют качественное сходство, поэтому относительный заряд капель в безэмиссионном режиме можно вычислить по аналогии с аналитической моделью при помощи модели сферического конденсатора. Однако расстояние между обкладками такого конденсатора в общем случае не равно радиусу Дебая и его значение необходимо вычислить точнее. Соответствующее расстояние имеет смысл эффективной ширины слоя $L_{\text {eff. }}$ Из сравнения кривых, обозначенных на рис. 4, $a$ штриховой и штрихпунктирной линиями можно найти аппроксимирующее 

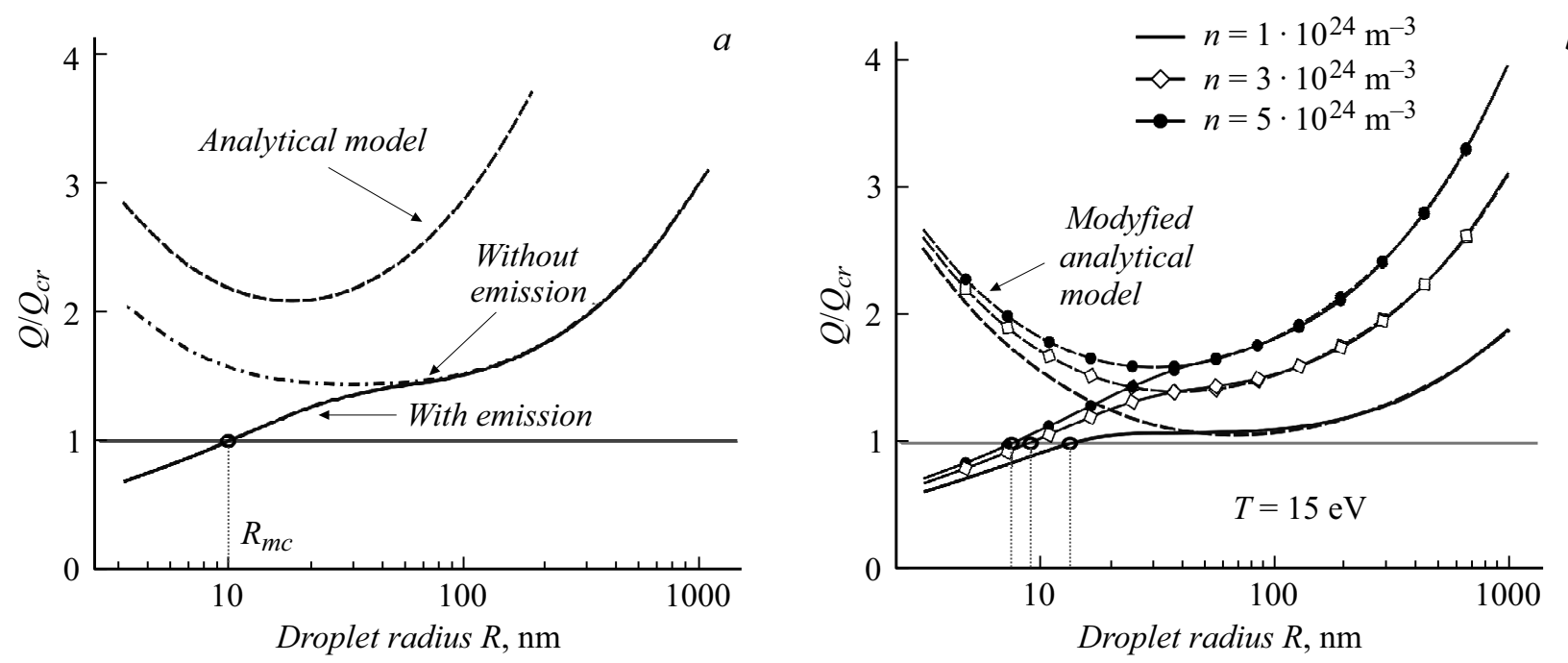

Рис. 4. Результаты расчета зависимости относительного заряда капли от ее радиуса. $a-$ сравнение результатов, полученных с использованием различных моделей. Параметры расчета: плотность плазмы $n=3 \cdot 10^{24} \mathrm{~m}^{-3}$, температура электронов $T_{e}=15 \mathrm{eV}$, работа выхода $w=5.0 \mathrm{eV}$, температура поверхности $T_{s}=3000 \mathrm{~K}$. $b-$ сравнение результатов расчета, полученных с использованием модифицированной аналитической модели (штриховые кривые) и с использованием численной модели (сплошные кривые) для различных плотностей плазмы. Параметры расчета: температура электронов $T_{e}=15 \mathrm{eV}$, работа выхода $w=5.0 \mathrm{eV}$, температура поверхности $T_{s}=3000 \mathrm{~K}$.

выражение для эффективной ширины слоя, которое имеет вид

$$
L_{\mathrm{eff}} \approx R_{D}\left(c_{1} \exp \left(-\frac{c_{2} R}{R_{D}}\right)+c_{3}\right)^{-1},
$$

где константы принимают следующие значения: $c_{1}=0.1$, $c_{2}=0.007, c_{3}=0.364$. Отметим, что для крупных капель эффективная ширина слоя лежит в диапазоне 2-3 радиусов Дебая. Используя далее выражение (16) и делая замену $R_{D} \rightarrow L_{\mathrm{eff}}$ с использованием (17), мы получаем формулу для расчета относительного заряда капли в рамках модифицированной аналитической модели

$$
\frac{Q}{Q_{c r}} \approx \frac{\sqrt{\varepsilon_{0} R}}{4 \sqrt{\alpha}} \frac{k T_{e}}{e} \ln \left(\frac{m_{i}}{m_{e}}\right)\left[\frac{1}{R}+\frac{1}{L_{\mathrm{eff}}}\right] .
$$

Таким образом, крупные капли металлов с радиусом больше $100 \mathrm{~nm}$ заряжаются в плазме в безэмиссионном режиме, и для вычисления относительного заряда капли можно использовать модифицированную аналитическую модель (17), (18). Проведем анализ применимости модифицированной аналитической модели с точки зрения процесса каскадного электродиспергирования.

Для определения области применимости модифицированной аналитической модели на рис. $4, b$ приведено ее сравнение с численным расчетом, проведенным для различных плотностей плазмы, по модели, в которой учитывается термополевая эмиссия электронов (сплошные линии). В первую очередь необходимо отметить, что влияние термополевой эмиссии приводит к снижению относительного заряда нанокапель радиусом около $10 \mathrm{~nm}$. Радиус капель, при котором относительный заряд равен единице, мы обозначим $R_{m c}$. Этот радиус имеет смысл минимального радиуса капель, при котором работает эффект непрерывной подзарядки капель до предела Рэлея в процессе каскадного деления. Другими словами, если радиус капель равен $R_{m c}$, то происходит их последнее каскадное деление.

Кроме этого, как видно из рис. 4, $b$, график относительного заряда капель, вычисленный по модифицированной аналитической модели, имеет точку минимума, причем кривая, описывающая результаты численного расчета, начинает существенно отличаться от аналитической кривой вблизи этой точки. Поэтому, если вычисленный по формуле (18) относительный заряд капли $Q / Q_{R}$ в точке минимума меньше единицы, то значение можно вычислить в рамках модифицированной аналитической модели. Для этого необходимо приравнять выражение в правой части (18) к единице и решить полученное уравнение. Сравнение результатов расчетов радиуса $R_{m c}$, полученных в рамках численной модели с учетом эмиссии электронов и модифицированной аналитической модели для различных плотностей плазмы, позволяет получить критерий применимости аналитической модели (18) для процесса каскадного электродиспергирования.

График зависимости минимального радиуса капли от плотности плазмы $R_{m c}(n)$ представлен на рис. 5, $a$. Расчет минимального радиуса проводился для случая двух температур плазмы: $T_{e}=10$ и $15 \mathrm{eV}$. Сплошная кривая показывает результаты расчета в рамках численной модели с учетом термополевой эмиссии, маркированная кривая — в рамках аналитической модели (18). Как видно из рисунка, аналитическая модель корректно описывает минимальный радиус вплоть до некоторого порогового значения плотности $n_{t h}$. При плотности плазмы 

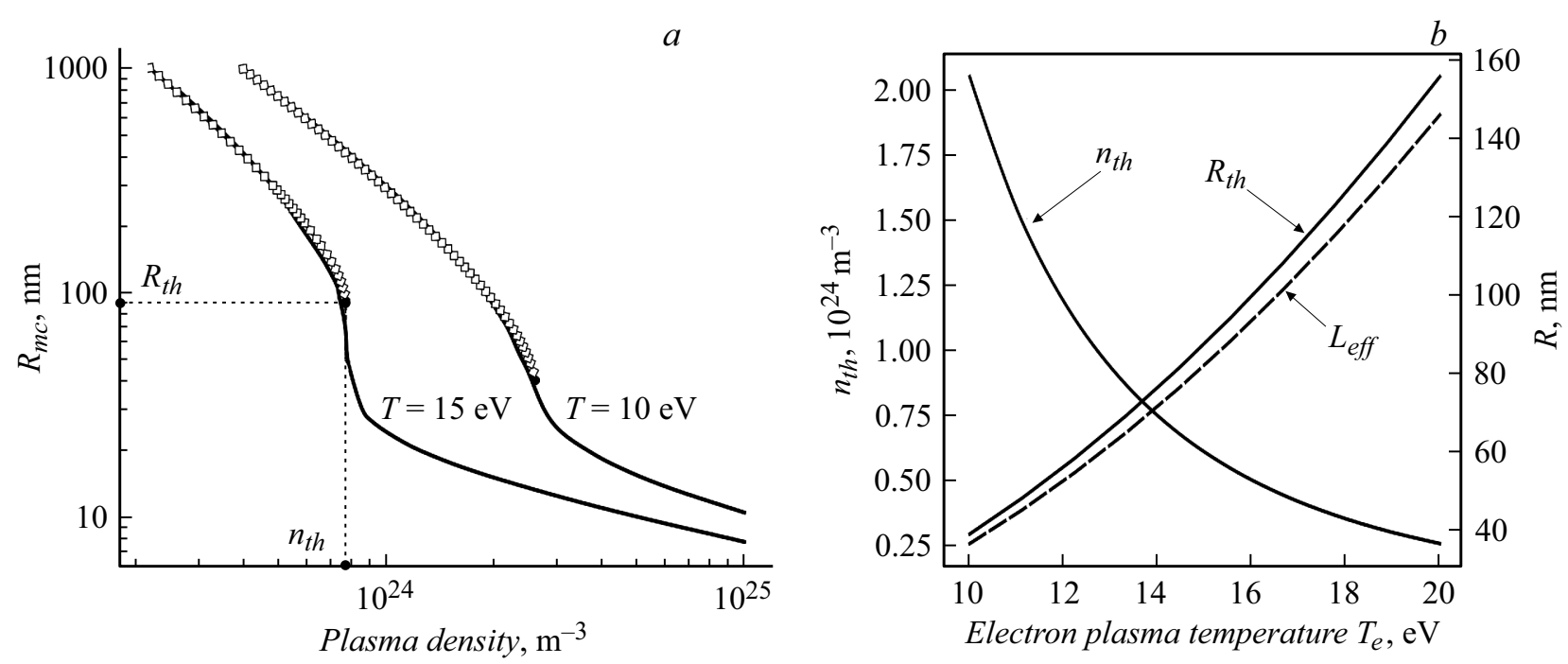

Рис. 5. $a-$ зависимость минимального радиуса от плотности плазмы. Работа выхода $w=5.0 \mathrm{eV}$, температура поверхности $T_{s}=3000 \mathrm{~K} . b-$ зависимость предельных параметров от температуры плазмы.

$n<n_{t h}$ заряд капли можно рассчитать без учета эмиссии, используя аналитическую формулу (18). Однако расчет заряда капли при плотности $n>n_{t h}$ необходимо проводить уже с учетом термополевой эмиссии в полном объеме.

Пороговой плотности $n_{t h}$ соответствует некоторый минимальный радиус $R_{t h}$, который мы также будем называть пороговым. Этот радиус показывает минимальный размер капель, которые могут быть заряжены в безэмиссионном режиме до предела Рэлея. Замечательным свойством этих величин является то, что они могут быть определены при помощи модифицированной аналитической модели, т.е. не зависят от таких параметров материала как работа выхода и температура поверхности. Другими словами, пороговые параметры зависят только от температуры плазмы.

Графики зависимостей $n_{t h}\left(T_{e}\right)$ и $R_{t h}\left(T_{e}\right)$ представлены сплошными линиями на рис. $5, b$. Как можно заметить из анализа графиков, при росте температуры от 10 до $20 \mathrm{eV}$, предельная плотность плазмы спадает с $2 \cdot 10^{24}$ до $4 \cdot 10^{23} \mathrm{~m}^{-3}$, а пороговый радиус увеличивается от 40 до $160 \mathrm{~nm}$. Более точный смысл порогового радиуса можно раскрыть, если сравнить его с эффективной шириной слоя $L_{\mathrm{eff}}$, график которой также приведен на рис. $5, b$ штриховой линией. Как можно заметить, эти величины очень близки при рассматриваемых электронных температурах плазмы. Это позволяет сделать вывод о том, что эмиссионный режим зарядки начинается, если радиус капли становится существенно меньше эффективной ширины слоя.

Тем не менее для задачи деления металлических нанокапель наиболее важен именно второй диапазон, так как именно в нем происходит зарядка до предела Рэлея капель нанометровых масштабов.

Зависимость минимального радиуса от температуры плазмы для капель нанометрового размера представлена на рис. 6, $a$. Сравнение графиков $R_{m c}\left(T_{e}\right)$ для различных значений плотности плазмы показывает, что минимальный радиус монотонно спадает при увеличении температуры, причем скорость убывания радиуса зависит от плотности плазмы.

Влияние параметров материала на радиус $R_{m c}$ также наблюдается только в эмиссионном режиме зарядки, т.е. при плотности плазмы $n>n_{t h}\left(T_{e}\right)$. При этом расчеты показывают, что минимальный радиус капли существенно зависит только от значений работы выхода металла и температуры поверхности капли, влиянием же энергии Ферми металла можно в общем случае пренебречь.

Графики зависимости величины минимального радиуса от работы выхода металла, построенные в рамках численной модели для значений температуры поверхности $T_{s}=3000$ и $4000 \mathrm{~K}$, представлены на рис. $6, b$ маркированными кривыми. Как видно из этих графиков, температура поверхности катастрофически влияет на минимальный радиус капли. Столь высокое различие между кривыми объясняется в первую очередь существенным вкладом надбарьерного потока электронов (температурной составляющей эмиссии) в общий эмиссионный поток.

Для сравнения на рис. $6, b$ приведена сплошная кривая, соответствующая формуле $R_{m c} \approx 8 \cdot 10^{-18} \alpha \varepsilon_{0}^{-1} w^{-3}$, представленной в работе [12]. В этой работе при расчете эмиссии электронов учитывался только поток туннелировавших электронов с поверхности холодного металла, т. е. полевая эмиссия, вычисленная по формуле ФаулераНордгейма, и для вывода выше приведенной формулы использовались приближенные аналитические методы решения нелинейного алгебраического уравнения. Как видно из сравнения кривых, эта формула дает сильно заниженную величину параметра $R_{m c}$.

Причина такого расхождения становится ясной при сравнении этих кривых со штриховой кривой, которая 

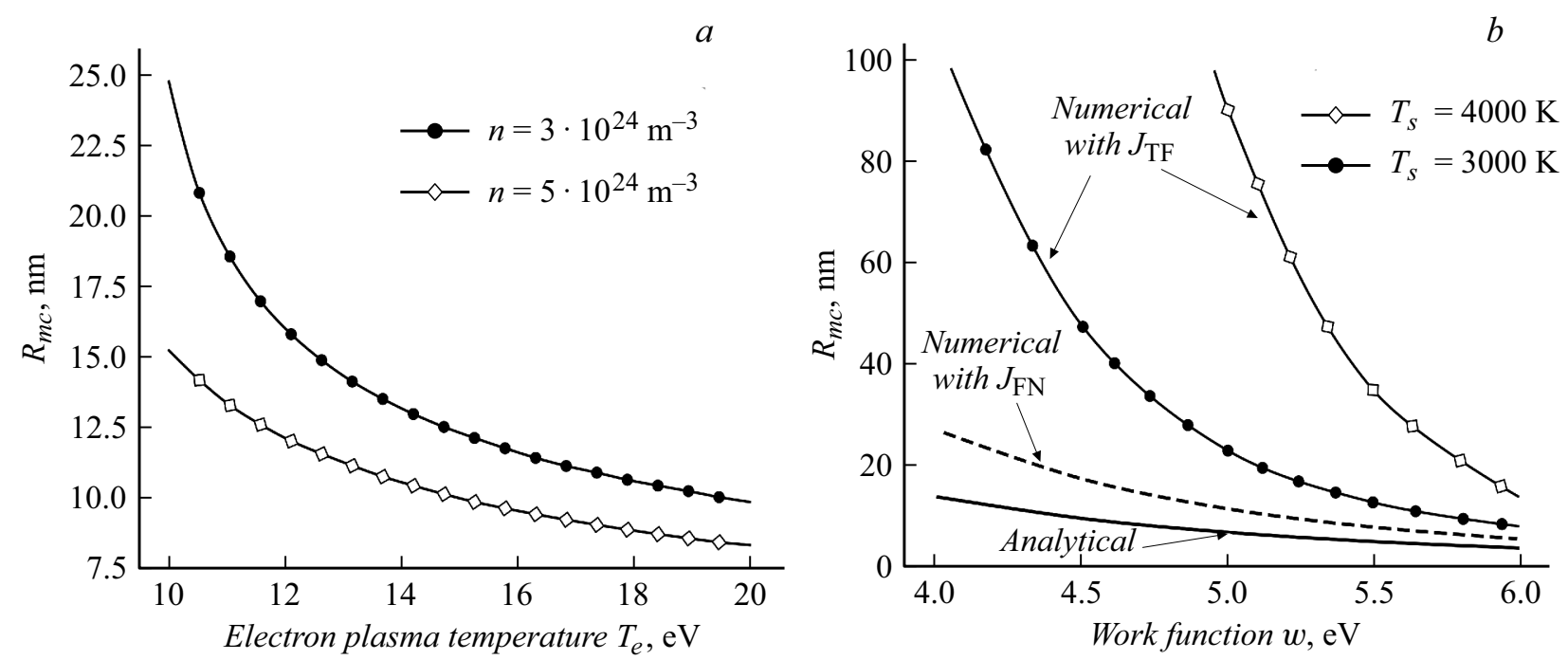

Рис. 6. $a-$ зависимость минимального радиуса от электронной температуры плазмы. Работа выхода $w=5.0 \mathrm{eV}$, температура поверхности $T_{s}=3000 \mathrm{~K} . b-$ зависимость минимального радиуса от работы выхода металла. Плотность плазмы $n=3 \cdot 10^{24} \mathrm{~m}^{-3}$, температура электронов $T_{e}=15 \mathrm{eV}$.

получена по результатам расчета в рамках численной модели с использованием формулы Фаулера-Нордгейма. Как следует из этого сравнения, основной причиной расхождения является пренебрежение в [12] влиянием температуры поверхности капель на интенсивность эмиссионного потока, в то время как использование приближенного метода решения уравнения оказалось не так существенно.

\section{Заключение}

Разработанная численная модель зарядки капель в плотной плазме, позволяющая существенно точнее описывать зависимость заряда капли от параметров материала и плазмы, является важным составным элементом процесса разработки новых технологий формирования аморфных металлических наноструктур. Необходимость использования представленной модели при проведении таких разработок, определяется тем, что в ней реализовано совмещение детального описания термополевой эмиссии электронов с поверхности капель с корректным расчетом распределения потенциала в плазменном слое, формирующимся на границе капель и плазмы, что значительно расширяет границы применимости этой модели.

Результаты проведенных расчетов показали, что процесс зарядки капель в плазме можно достаточно четко разделить на два режима: эмиссионный и безэмиссионный. Крупные капли металлов заряжаются в плазме в безэмиссионном режиме, и для вычисления относительного заряда капли можно использовать полученную в работе модифицированную аналитическую модель (17), (18). Модифицированная аналитическая модель может применяться для анализа процесса каскадного электродиспергирования, только для капель, радиус которых больше эффективной ширины слоя (17), которая составляет 2-3 радиуса Дебая.

Результаты расчетов зарядки капель в эмиссионном режиме показали, что минимальный радиус капель, при котором происходит непрерывное каскадное деление, монотонно спадает при увеличении температуры плазмы, причем скорость убывания радиуса зависит от плотности плазмы. При анализе результатов расчетов зарядки капли в эмиссионном режиме было показано, что минимальный радиус капли существенно зависит только от значений работы выхода металла и температуры поверхности капли, влиянием же энергии Ферми металла можно в общем случае пренебречь. Температура поверхности капель существенно влияет на величину минимального радиуса, что объясняется в первую очередь существенным вкладом надбарьерного потока электронов (температурной составляющей эмиссии) в общий эмиссионный поток. Важно отметить, что задача определения температуры поверхности нанокапли в плазме лазерного факела является открытой и ключевой для повышения точности моделей электродиспергирования металлов.

Помимо этого, другим важным вопросом является определение времени каскадного электродиспергирования капель. Модель развития неустойчивости Рэлея для заряженной капли в вакууме, представленная в работе [33], показывает, что характерное время развития неустойчивости падает с уменьшением радиуса капель, поэтому время каскадного деления капель в первую очередь определяется временем развития неустойчивости на крупной материнской капле. Однако вопрос применимости модели [33] для крупных капель в плазме лазерного факела, радиус которых сравним с шириной слоя, остается открытым, так как деформирующее каплю давление определяется распределением электрического 
поля в слое вблизи поверхности капли, и может существенно отличаться от распределения давления на поверхности заряженной капли в вакууме. Поэтому оценка времени каскадного деления при лазерном электродиспергировании также требует дальнейших подробных исследований.

\section{Конфликт интересов}

Авторы заявляют, что у них нет конфликта интересов.

\section{Список литературы}

[1] К. Судзуки, Х. Фудзимори, К. Хасимото. Аморфные металлы (Металлургия, М., 1987)

[2] T. Gloriant. J. Non-Crystalline Solids, 316 (1), 96 (2003). DOI: $10.1016 / \mathrm{S} 0022-3093(02) 01941-5$

[3] C. Suryanarayana. Inoue A. Bulk metallic glasses (CRC press, 2017)

[4] M. Avrami. J. Chem. Phys., 7 (12), 1103 (1939). DOI: $10.1063 / 1.1750380$

[5] И.В. Золотухин. Физические свойства аморбных металлов (Металлургия, М., 1986)

[6] H.A. Davies, J.B. Hull. J. Mater. Sci., 11 (2), 215 (1976). DOI: $10.1007 / \mathrm{BF} 00551430$

[7] C.D. Hendricks, J M. Schneider. American J. Phys., 31 (6), 450 (1963). DOI: 10.1119/1.1969579

[8] А.И. Григорьев, С.О. Ширяева. ЖТФ, 61 (3), 19 (1991).

[9] S.A. Ryce, D.A. Patriarche. Canad. J. Phys., 43 (12), 2192 (1965). DOI: 10.1139/p65-213

[10] H.M.A. Elghazaly G.S., Castle P. IEEE Trans. Industry Applications, 25 (1), 48 (1989). DOI: 10.1109/28.18868

[11] V.M. Kozhevin, D.A. Yavsin, V.M. Kouznetsov, V. Busov, V.M. Mikushkin, S.Yu. Nikonov, S.A. Gurevich, A. Kolobov. J. Vacuum Sci. Technol. B, 18 (3), 1402 (2000). DOI: $10.1116 / 1.591393$

[12] T.N. Rostovshchikova, E.S. Lokteva, E.V. Golubina, K.I. Maslakov, S.A. Gurevich, D.A. Yavsin, V.M. Kozhevin. Adv. Size-Selected Catalysts Prepared by Laser Electrodispersion. In Advanced Nanomaterials for Catalysis and Energy (Elsevier, 2019), p. 61.

[13] В.Г. Дудников, А.Л. Шабалин. Электрохимические источники ионных пучков (ИЯФ СО ФН СССР, Новосибирск, 1987)

[14] A.P. Bakhtinov, V.B. Boledzyuk, Z.D. Kovalyuk, Z.R. Kudrynsky, O.S. Lytvyn, A.D. Shevchenko. Phys. Solid State, 55 (6), 1148 (2013). DOI: $10.1134 / \mathrm{S} 1063783413060048$

[15] J.F. Mahoney, S. Taylor, J. Perel. IEEE Transactions on Industry Applications, (2), 197 (1987). DOI: 10.1109/TIA.1987.4504894

[16] C. Bartoli, H. Von Rohden, S.P. Thompson, J. Blommers. Vacuum, 34 (1-2), 43 (1984). DOI: $10.1016 / 0042-207 X(84) 90105-2$

[17] Y.E. Kim, M. Rabinowitz, G.S. Chulick, R.A. Rice. Modern Phys. Lett. B, 5 (06), 427 (1991). DOI: $10.1142 / \mathrm{S} 0217984991000502$

[18] V.M. Kozhevin, D.A. Yavsin, I.P. Smirnova, M.M. Kulagina, S.A. Gurevich. Phys. Solid State, 45 (10), 1993 (2003). DOI: $10.1134 / 1.1620108$
[19] D.S. Ilyushenkov, V.I. Kozub, D.A. Yavsin, V.M. Kozhevin, I.N. Yassievich, T.T. Nguyen, E.H. Bruck, S.A. Gurevich. J. Magn. Magn. Mater., 321 (5), 343 (2009).

DOI: $10.1016 /$ j.jmmm.2008.09.024

[20] T.N. Rostovshchikova, V.V. Smirnov, V.M. Kozhevin, D.A. Yavsin, M.A. Zabelin, I.N. Yassievich, S.A. Gurevich. Appl. Catalys. A: General, 296 (1), 70 (2005). DOI: $10.1016 /$ j.apcata.2005.08.032

[21] A.Y. Khairullina, T.V. Ol'shanskaya, V.A. Babenko, V.M. Kozhevin, D.A. Yavsin, S.A. Gurevich. Opt. Spectr., 98 (1), 96 (2005). DOI: 10.1134/1.1858046

[22] В.М. Вержбицкий. Основы численных методов (Высшая школа, М., 2005)

[23] P.M. Chung, L. Talbot, K.J. Touryan. Electric Probes in Stationary and Flowing Plasmas: Theory and Application (Springer Science \& Business Media, 2013)

[24] В.А. Рожанский. Теория плазмы (Лань, СПб., 2012)

[25] Н. Ашкрофт, Н. Мермин. Физика твердого тела (Мир, M., 1979)

[26] А.Б. Петрин. ЖЭТФ, $136(2), 369$ (2009).

[27] J.T. Holgate, M. Coppins. Phys. Rev. Appl., 7 (4), 044019 (2017). DOI: 10.1103/PhysRevApplied.7.044019

[28] O.W. Richardson. The Emission of Electricity from Hot Bodies (Longmans, 1921)

[29] R.H. Fowler, L. Nordheim. Proc. R. Soc. A, 119 (781), 173 (1928). DOI: 10.1098/rspa.1928.0091

[30] R.G. Forbes. Appl. Phys. Lett., 89 (11), 113122 (2006). DOI: $10.1063 / 1.2354582$

[31] E.L. Murphy, R.H. Good Jr. Phys. Rev., 102 (6), 1464 (1956). DOI: $10.1103 /$ PhysRev.102.1464

[32] И.С. Григорьев, Е.3. Мейлихов. Физические величины (Энергоатомиздат, М., 1991)

[33] С.О. Ширяева. Письма в ЖТФ, 26 (4), 5 (2000). 